\title{
Effect of quadrivalent HPV vaccination on HPV related disease in women treated for cervical or vulvar/vaginal disease
}

In the fourth paragraph of this Editorial by Jane J Kim (BMJ 2012;344:e1544, doi:10.1136/bmj.e1544), it should be noted that disease prevented may have also resulted from an

anamnestic response generated by the vaccine in women who had cleared prior vaccine-type infections. This effect has also been observed in previous studies.

Cite this as: BMJ 2012;344:e2501

๑ BMJ Publishing Group Ltd 2012 